Pacific Journal of Mathematics

GROUPS WHICH ARE MINIMAL WITH RESPECT TO 


\title{
GROUPS WHICH ARE MINIMAL WITH RESPECT TO NORMALITY BEING INTRANSITIVE
}

\author{
DEREK J. S. ROBINSON
}

\begin{abstract}
A $\mathfrak{T}$-group is a group in which normality is transitive and a minimal non- $\mathfrak{T}$-group is a group which is not a $\mathfrak{T}$-group but all of whose proper subgroups are $\mathfrak{T}$-groups. In this note all the finite minimal non- $\mathfrak{T}$-groups are determined and it is shown that this classification also accounts for all the minimal nonI-groups which are either locally finite or 2-groups.
\end{abstract}

1. $\mathfrak{T}$-groups and non- $\mathfrak{T}$-groups. Let $\mathfrak{I}$ denote the class of all groups in which normality is a transitive relation: in other words a group $G$ belongs to $\mathfrak{I}$ if and only if $H \triangleleft K \triangleleft G$ always implies that $H \triangleleft G$. $\overline{\mathfrak{I}}$ denotes the narrower class of all groups $G$ such that $H \triangleleft K \triangleleft L \leqq G$ implies that $H \triangleleft L$. A group belonging to a class of groups $\mathfrak{X}$ is called an $\mathfrak{X}$-group and other groups are called non- $\mathfrak{X}$ -

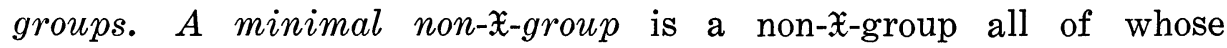
proper subgroups are $\mathfrak{X}$-groups.

The problem of determining all the finite minimal non- $\mathfrak{X}$-groups has been studied by several authors when $\mathfrak{X}$ is the class of abelian, nilpotent, supersoluble or soluble groups. Our principal object here is the classification of all the finite minimal non-T-groups or, what is obviously the same thing, the finite minimal non- $\overline{\mathfrak{T}}$-groups. Although the nature and even the existence of infinite minimal non-T-groups is problematic, our classification does account for all minimal non- $\mathfrak{I}-$ groups which are locally finite or 2-groups or SI-groups (in the sense of Kuroš [5], vol. 2, §57).

The finite minimal non-T-groups. Such groups are all soluble and it turns out that they fall naturally into seven types, which will now be listed under four headings, beginning with the primary groups.

I. The primary groups.

(a) The generalized quaternion group of order 16.

(b) $\left\langle x, y: x^{p^{m}}=1=y^{p^{n}}, x^{-1} y x=y^{1+p^{n-1}}\right\rangle$ where $p$ is any prime, $m>0$ and $n>1$.

(c) $\left\langle x, y, z: z=[x, y], 1=x^{p^{m}}=y^{p^{n}}=z^{p}=[x, z]=[y, z]\right\rangle$ where $p$ is any prime, $m>0$ and $n>0$.

II. The nonprimary groups with quaternion subgroups. The semi-direct product of a quaternion group of order 8 with a cyclic group of order $3^{m}(m>0)$ which induces an automorphism permuting cyclically the three maximal subgroups of the quaternion group.

III. The supersoluble, nonprimary groups. Let $p$ and $q$ be 
primes such that $p \equiv 1 \bmod q$ and let $q^{f}$ divide $p-1$ where $f>0$. $\langle a\rangle \times\langle b\rangle$ is an elementary abelian $p$-group of order $p^{2}$ and $X=\langle x\rangle$ is a cyclic group of order $q^{m}$ where $m \geqq f$. Let $\xi$ be a primitive $q^{f}$ th root of unity in $G F(p)$.

The semi-direct product of $\langle a\rangle \times\langle b\rangle$ with $X$ in which $x^{-1} a x=a^{\xi}$ and $x^{-1} b x=b^{\eta}$ where $\eta=\xi^{1+k q^{f-1}}$ and $0<k<q$.

IV. The nonsupersoluble groups without quaternion subgroups.

(a) Let $p$ and $q$ be distinct primes such that $p \not \equiv 1 \bmod q$ and let $F$ be the field obtained by adjunction to $G F(p)$ of $\xi$, a primitive $q$ th root of unity. Let $A$ be the additive group of $F$ and let $X=$ $\langle x\rangle$ be a cyclic group of order $q^{m}$ where $m>0$.

The semi-direct product of $A$ with $X$ where $x$ induces in $A$ the automorphism $a \rightarrow a \xi(a \in A)$.

(b) Let $p$ and $q$ be primes such that $p \equiv 1 \bmod q$ and let $q^{f-1}$ be the highest power of $q$ dividing $p-1 . A$ is an elementary abelian $p$-group of order $p^{q}$ with basis $\left\{a_{0}, \cdots, a_{q-1}\right\}$ and $X=\langle x\rangle$ is a cyclic group of order $q^{m}$ where $m \geqq f$. Let $\lambda$ be a primitive $q^{f-1}$ th root of unity in $G F(p)$.

The semi-direct product of $A$ with $X$ where $x$ induces in $A$ the automorphism in which $a_{i} \rightarrow a_{i+1},(i=0, \cdots, q-2)$, and $a_{q \sim 1} \rightarrow a_{0}^{\lambda}$.

Thus our principal result is precisely

THEOREM 1. A finite group is a minimal non-T-group if and only if it is of one of the above seven types.

It is easy to verify that each finite minimal non-T-group is a 2-generator, 3-step soluble group with order divisible by at most 2 primes. Consequently using the local theorem for the class $\mathfrak{I}$ ([9], p. 22, Corollary 2) we obtain at once

THeorem 2. A locally finite group is a $\overline{\mathfrak{T}}$-group if and only if each of its 2-generator, 3-step soluble subgroups with order divisible by at most 2 primes is a $\mathfrak{I}$-group.

The basis for the determination of the finite minimal non- $\mathfrak{T}-$ groups is the following result $(R)$, which was obtained in a previous paper ([11], Th. 1). A finite group is said to satisfy the p-normalizer condition if every subgroup of a Sylow $p$-subgroup is normal in the normalizer of that Sylow $p$-subgroup.

(R) A finite group which satisfies the p-normalizer condition for all primes $p$ is a soluble $\mathfrak{I}$-group. ${ }^{1}$

1 The $p$-normalizer condition is equivalent to the property that every cyclic $p$-subgroup is pronormal ([11], p. 936), so (R) may be restated in terms of pronormality. A special case of this form of $(R)$ was later given by Peng in [7]. 
Notation. If $X$ and $Y$ are subgroups of a group, $C_{X}(Y)$ and $N_{X}(Y)$ are respectively the centralizer and normalizer of $Y$ in $X$. $\langle S\rangle$ is the subgroup generated by the subset $S$. If $S$ and $T$ are subsets, $S^{T}$ is the subgroup generated by all the conjugates $s^{t}=t^{-1} s t$, and $[S, T]$ is the subgroup generated by all the commutators $[s, t]=$ $s^{-1} t^{-1} s t=s^{-1} s^{t},(s \in S, t \in T)$.

2. Proof of Theorem 1. The verification of the fact that each of the above seven types of groups is a minimal non-T-group is routine and details will not be given. Notice that it is sufficient to verify that in each case the group is not a $\mathfrak{I}$-group but every maximal subgroup is a $\mathfrak{T}$-group: this is in view of a theorem of Gaschuitz according to which a finite soluble $\mathfrak{I}$-group is a $\overline{\mathfrak{I}}$-group ([2]). We proceed now to determine the structure of finite minimal non-T-groups.

The primary case. Let $G$ be a finite minimal non-I-group and assume that $G$ is a $p$-group where $p$ is a prime. Then every proper subgroup of $G$ is either abelian or hamiltonian. Now the finite minimal nonabelian groups have been determined by Miller and Moreno ([6]) and, in somewhat greater detail, by Rédei ([8]). The $p$-groups among these are of three kinds, namely type $\mathrm{I}(\mathrm{b})$, type $\mathrm{I}(\mathrm{c})$ and the quaternion group of order 8 , which is, of course, ineligible in our case.

Thus we can suppose that $G$ has a nonabelian maximal subgroup $A$. Clearly $p=2$ and $A$ is a normal hamiltonian subgroup of index 2 in $G$ : let

$$
A=Q \times E
$$

where $Q$ is a quaternion group of order 8 and $E$ is an elementary abelian 2-group. For any $x \in G \backslash A$,

$$
G=\langle x, A\rangle \text { and } x^{2} \in A .
$$

Clearly $Q^{\prime} \times E \triangleleft G$, so $\left\langle x, Q^{\prime} \times E\right\rangle$ is a cyclic extension of an elementary abelian 2-group. Since $Q$ does not have this structure, $\left\langle x, Q^{\prime} \times E\right\rangle \neq G$ and $\left\langle x, Q^{\prime} \times E\right\rangle \in \mathfrak{T}$; but $Q^{\prime} \times E$ is an elementary abelian 2-group, so

$$
[E, x]=1 \text { and } E \triangleleft G \text {. }
$$

Of course $A / E \cong Q$ and $x$ induces in $A / E$ an automorphism of order 1,2 or 4 , so $x$ must leave invariant at least one of $\langle i E\rangle,\langle j E\rangle$ and $\langle k E\rangle$, where $\langle i\rangle,\langle j\rangle$ and $\langle k\rangle$ are the three maximal subgroups of $Q$ and the usual relations $i^{2}=j^{2}=k^{2}, i j=k, j k=i$ and $k i=j$ hold. Suppose first that $x^{2} \notin Q^{\prime} \times E$. If, say, $x^{2}=i e$ where $e \in E$, let 
$i^{*}=i e, j^{*}=j e$ and $k^{*}=k$ : then $Q^{*}=\left\langle i^{*}, j^{*}, k^{*}\right\rangle \cong Q$ and $A=$ $Q^{*} \times E$, so we may assume that $x^{2}=i$. Now $x$ certainly leaves $\langle i E\rangle$ invariant: if $x$ also leaves $\langle j E\rangle$ invariant, then $x^{2}=i$ centralises $j E$, which is not the case. Hence $x$ leaves $\langle i E\rangle$ invariant and interchanges $\langle j E\rangle$ and $\langle k E\rangle$. Since $x^{2}=i$, we can suppose that for example

$$
j^{x}=k^{-1} e \text { and } k^{x}=j e
$$

for some $e \in E$. If $e \neq 1,|G|=16 .|E|>16$. Let $H=\langle x j, i\rangle$. Since $\langle i, e\rangle \triangleleft G$ and $(x j)^{2}=x^{2} k^{-1} e j=i^{2} e,|H|=4 \times 2 \times 2=16$ and hence $H \in \mathfrak{T}$. Therefore $(x j)^{i}=x j^{-1} \in\langle x j\rangle$ and $x j^{-1}=(x j)^{3}=x j^{3} e$, which implies that $e=1$. By this contradiction $e=1$ and $j^{x}=k^{-1}$ and $k^{x}=j$. Let $K=\langle x, j\rangle$ : then $x^{j}=x k j=x i^{-1}=x^{-1}$, and $x^{4}=j^{2}$ and $x^{8}=1$. Hence $K$ is a generalized quaternion group of order 16 . Since $K \notin \mathfrak{I}, G=K$. If on the other hand $j^{x}=k e$ and $k^{x}=j^{-1} e$ where $e \in E$, a similar argument shows that $e=1$ and $j^{x}=k$ and $k^{x}=j^{-1}$. Hence $x^{j}=x^{3}$ and $\left(x j^{-1}\right)^{2}=1$, by a simple calculation. The group $L=\left\langle x^{2}, x j^{-1}\right\rangle$ is therefore a dihedral group of order 8 . Hence $G=L$, which is impossible since $G$ has order $\geqq 16$.

Suppose now that $x^{2} \in Q^{\prime} \times E$. Assume that $x$ interchanges $\langle j E\rangle$ and $\langle k E\rangle$ and let $z=x j$ : then $z^{2} \equiv x^{2} k j \equiv i \bmod Q^{\prime} \times E$, so $z^{2} \notin Q^{\prime} \times E$, while $G=\langle z, A\rangle$. Hence $G$ is a generalized quaternion group of order 16. Thus we can assume that $x$ fixes each of $\langle i E\rangle,\langle j E\rangle$, and $\langle k E\rangle$. Suppose for example that $i^{x}=i^{-1} e, j^{x}=j^{-1} f$ and $k^{x}=k g$ where $e, f, g \in E$ and $e f g=1$. Set $u=x k$ : then $i^{u}=i e, j^{u}=j f$ and $k^{u}=k g$ while $G=\langle u, A\rangle$ and $u^{2}=x^{2} k^{2} g \in Q^{\prime} \times E$. Hence we may assume that $i^{x}=i e, j^{x}=j f$ and $k^{x}=k g$ where $e f g=1$. It is immediate that $\langle x, i, e\rangle \neq G$, so that $\langle x, i, e\rangle \in \mathfrak{I}$ and $i^{x}=i e \in\langle i\rangle$. Therefore $e=1$ and similarly $f=g=1$; hence $[Q, x]=1$. If $x^{2}=1$, $G=\langle x\rangle \times Q \times E$, which is hamiltonian. Hence $x^{2} \neq 1$ and consequently $(x i)^{j}=x i^{-1}$ differs from both $x i$ and $x^{3} i^{3}$. Therefore $\langle x i, j\rangle \notin \mathfrak{I}$ and $G=\langle x i, j\rangle$. However $Q=(Q \cap\langle x i\rangle)\langle j\rangle$, so $Q \cap\langle x i\rangle$ has order 4 and $x i \in Q$. Hence $x \in Q$ and therefore this case cannot arise.

The nonprimary case. Let $G$ be a minimal non-T-group of composite order. By the result $(\mathrm{R})$ there is a prime $p$ and a Sylow $p$-subgroup $P$ of $G$ with a subgroup which is not normal in $N_{G}(P)$. Since every subgroup of $P$ is subnormal in $N_{G}(P), N_{G}(P)=G$ and $P \triangleleft G$. By the Schur-Zassenhaus Theorem $P$ has a complement in $G$, say $X$. Since $1<P<G$, both $P$ and $X$ belong to $\mathfrak{I}$ and, of course, they have relatively prime orders. Hence by Lemma 5.2.2. of [9] there is an element $x$ in $X$ with prime power order $q^{m}$ which does not induce a power automorphism in $P$, i.e., which does not 
leave every subgroup of $P$ invariant. $\langle x, P\rangle \notin \mathfrak{T}$, so $G=\langle x, P\rangle$ and $X=\langle x\rangle$.

Suppose that $P$ is hamiltonian-so $p=2$-and that

$$
P=Q \times E
$$

where $Q$ is a quaternion group of order 8 and $E$ is an elementary abelian 2-group. Since $\left\langle x, Q^{\prime} \times E\right\rangle \neq G$, it follows that

$$
[E, x]=1 \text {. }
$$

Once again let $\langle i\rangle,\langle j\rangle$ and $\langle k\rangle$ be the three maximal subgroups of $Q$. Since $x$ has odd order, it cannot centralize $P / E$. Moreover $P / E \cong Q$ and $x$ has prime power order $q^{m}$, so $q=3$ and $x$ permutes $\langle i E\rangle$, $\langle j E\rangle$ and $\langle k E\rangle$ cyclically. If for example

$$
i^{x}=j^{-1} e, j^{x}=k^{-1} f \text { and } k^{x}=i g
$$

where $e, f, g \in E$ and $e f g=1$, let $i^{*}=i^{-1}, j^{*}=j$ and $k^{*}=k^{-1}$. Then

$$
i^{*} j^{*}=k^{*}, j^{*} k^{*}=i^{*} \text { and } k^{*} i^{*}=j^{*}
$$

and

$$
\left(i^{*}\right)^{x}=j^{*} e,\left(j^{*}\right)^{x}=k^{*} f \text { and }\left(k^{*}\right)^{x}=i^{*} g .
$$

Thus we may assume that

$$
i^{x}=j e, j^{x}=k f \text { and } k^{x}=i g .
$$

Since $e f g=1, \bar{Q}=\langle i f, j g, k e\rangle \cong Q$ and $\bar{Q}$ is $\langle x\rangle$-invariant: therefore $\langle x, \bar{Q}\rangle$ is of type II. Since $\langle x, \bar{Q}\rangle \notin \mathfrak{T}, G=\langle x, \bar{Q}\rangle$.

From now on we suppose that $P$ is abelian. The first point to notice is that $P$ is elementary abelian. For let $P_{1}$ be the subgroup generated by all elements of order $p$ in $P$ and assume that $P_{1} \neq P$. Then $\left\langle x, P_{1}\right\rangle \neq G$, so $\left\langle x, P_{1}\right\rangle \in \mathfrak{T}$. Therefore $x$ induces a power automorphism in $P_{1}$. Hence there is a positive integer $r$, relatively prime to $p$, such that $a^{x}=a^{r}$ for all $a \in P$ (see [2], p. 88). Let $\alpha$ be the automorphism of $P$ induced by $x$ and let $\beta$ be the automorphism of $P$ in which $a \rightarrow a^{r}$. Then $\alpha \beta^{-1}$ is an automorphism of $P$ fixing each element of order $p$; consequently $\alpha \beta^{-1}$ has order equal to a power of $p$, say $p^{s}$. Obviously $\alpha \beta=\beta \alpha$, so $\alpha^{p^{s}}=\beta^{p^{s}} \in\langle\beta\rangle$. But $\alpha$ has order prime to $p$, so $\alpha \in\langle\beta\rangle$ and $\alpha$ is a power automorphism of $P$, which is impossible.

Since $P$ is an elementary abelian $p$-group, it may be regarded as a module over $\mathscr{R}$, the group algebra of $X$ over $G F(p)$. From now on $P$ will be written additively.

Assume first of all that $P$ is reducible. By Maschke's Theorem 
$P$ is a direct sum of irreducible modules $P_{1}, \cdots, P_{k}$ where $k>1$. Suppose that $k>2$. $\left\langle x, P_{2} \oplus \cdots \oplus P_{k}\right\rangle$ is a proper subgroup of $G$ so it belongs to $\mathfrak{I}$ and $x$ induces in $P_{2} \oplus \cdots \oplus P_{k}$ an automorphism $a \rightarrow a u$ for some integer $u$. Similarly $x$ induces in $P_{1} \oplus \cdots \oplus P_{k-1}$ an automorphism $a \rightarrow a v$ for some integer $v$. These automorphisms must agree on $P_{2}$, so $u \equiv v \bmod p$ and hence $x$ leaves every subgroup of $P$ invariant. By this contradiction $k=2$. Clearly each $P_{i}$ is cyclic as an additive group and $p \neq 2$. Let $q^{f}$ be the order of the automorphism of $P$ induced by $x$. If $P_{1}=\langle a\rangle$ and $P_{2}=\langle b\rangle$, then

$$
a x=a \xi \text { and } b x=b \eta
$$

where $\xi$ and $\eta$ are distinct $q^{f}$ th roots of unity in $G F(p)$, at least one, say $\xi$, being primitive. Then $0<f \leqq m$ and $q^{f} \mid p-1$. Since $\left\langle x^{q}, P\right\rangle \neq G, x^{q}$ induces a power automorphism in $P$ and

$$
\xi^{q}=\eta^{q} \text {. }
$$

If $\eta=1, \xi$ is a primitive $q$ th root of unity in $G F(p)$ and $G$ is of type III with $f=1$ and $\mathrm{k}=q-1$. Suppose $\eta \neq 1$, so that $\xi$ and $\eta$ both have order $q^{f}$. Then $\eta=\xi^{r}$ for some integer $r \not \equiv 1 \bmod q^{f}$. Now $\xi^{q}=\eta^{q}=\xi^{r q}$, so $r \equiv 1 \bmod q^{f-1}$ and we can assume that $r=$ $1+k q^{f-1}$ where $0<k<q$. Hence $G$ is again of type III.

Suppose now that $P$ is an irreducible $\mathscr{R}$-module and let $0 \neq a \in P$. Then $P=a \mathscr{R}$ and the map $r \rightarrow a r(r \in \mathscr{R})$ is a homomorphism of $\mathscr{R}$ (regarded as a right $\mathscr{R}$-module) onto $P$ : its kernel $K$ is a maximal ideal of $\mathscr{R}$ and $\mathscr{R} / K \cong P . F=\mathscr{R} / K$ is a finite field extension of $G F(p)$ obtained by adjoining $\xi=x+K$. Clearly $\xi$ is a primitive $q^{f}$ th root of unity over $G F(p)$, where $q^{f}$ is the order of the automorphism induced in $P$ by $x$. Let $G^{*}$ be the semi-direct product of the additive group of $F$ with $X$, where $x$ induces the automorphism $b \rightarrow b \xi(b \in F)$. Clearly $G \cong G^{*}$, so we can identify $G$ with $G^{*}$.

Since $\left\langle x^{q}, P\right\rangle \neq G, x^{q}$ induces a power automorphism in $P$ and $\lambda=\xi^{q}$ belongs to $G F(p)$. If $\lambda=1$, then $f=1, \xi$ is a primitive $q$ th root of unity over $G F(p)$ and $p \neq \equiv 1 \bmod q$. Hence $G$ is of type IVa. Assume that $\lambda \neq 1$, so $f>1, \lambda$ is a primitive $q^{f-1}$ th root of unity in $G F(p)$ and $p \equiv 1 \bmod q$. Suppose that $\lambda=\eta^{q}$ where $\eta$ belongs to $G F(p)$. Then $\xi$ and $\eta$ are elements of the same order in $F$. Hence $\xi \in\langle\eta\rangle$ and $\xi$ belongs to $G F(p)$. However this implies that $x$ induces a power automorphism in $P$. Hence $\lambda$ is not the $q$ th power of any element of $G F(p)$; thus the polynomial $t^{q}-\lambda$ is irreducible over $G F(p)$ (see [12], p. 179) and must therefore be the minimal polynomial of $\xi$. Let $\mathrm{a}_{i}=a \xi^{i}, i=0,1, \cdots, q-1$ : then $\left\{a_{0}, a_{1}, \cdots, a_{q-1}\right\}$ is a basis for $P$ over $G F(p)$ and $P$ has order $p^{q}$. Also $a_{i} x=a_{i+1}, \quad i=$ $0, \cdots, q-2$, and $a_{q-1} x=a_{0} \lambda$. Finally $\langle\lambda\rangle$ is the $q$-component of the 
multiplicative group of $G F(p)$, so $q^{f-1}$ is the highest power of $q$ dividing $p-1$ and $G$ is of type IVb.

3. Infinite minimal non-T-groups. Whether there are any infinite minimal non-I-groups we have not been able to decide: the following result represents our conclusions on this subject.

THEOREM 3. Let $G$ be a minimal non-I-group and suppose that $G$ has a series whose factors are either locally finite or 2-groups and whose terms are either ascendant or descendant in G. Then $G$ is finite.

Here the term "series" is used in the sense of P. Hall ([4]). A series in a group $G$ is a set of subgroups $\left\{\Lambda_{\sigma}, V_{\sigma}: \sigma \in \Sigma\right\}$ where $\Sigma$ is a linearly ordered set and the following properties hold: $V_{\sigma} \triangleleft \Lambda_{\sigma}$, $\Lambda_{\sigma} \leqq V_{\tau}$ if $\sigma<\tau$ and every nontrivial element of $G$ belongs to some $\Lambda_{\sigma} \backslash V_{\sigma}$ (c.f. "normal system" in Kuroš [5], §56).

We precede the proof with two observations about a minimal non-T-group $G$. Such a group is necessarily finitely generated, since otherwise every finitely generated subgroup of $G$ belongs to $\mathfrak{I}$ and $G$ belongs to $\mathfrak{I}$ by the local theorem for the class $\mathfrak{T}$. Also every subnormal subgroup $H$ of $G$ has subnormal index $\leqq 2$ : for if $H \neq G$, then $H^{G} \neq G$ and $H \triangleleft H^{G} \triangleleft G$.

Proof of Theorem 3. (a) Assume first that $G$ is a soluble minimal non-I-group. Then $G$ is finitely generated and every subnormal subgroup of $G$ has subnormal index $\leqq 2$, so by Theorem A of [10] $G$ has a normal finite subgroup with nilpotent factor group. If $G$ is infinite, by elementary results about finitely generated nilpotent groups $G$ has an infinite cyclic factor group, say $G / N$. Then $G=$ $\langle x, N\rangle$ where $x$ has infinite order. If $i>1,\left\langle x^{i}, N\right\rangle \neq G$ and $\left\langle x^{i}, N\right\rangle \in \mathfrak{T}$. Now $\left\langle x^{i}, N\right\rangle$ is finitely generated-either because it has finite index in $G$ or because $G$ satisfies the maximal condition on subgroups - and a finitely generated soluble $\mathfrak{I}$-group is finite or abelian ([9], Th. 3.3.1). Hence $\left[x^{i}, N\right]=1$ for all $i>1$ and thus $[x, N]=$ 1. Let $a \in N$ and $y=x a$. Then $G=\langle y, N\rangle$ and by the same argument $1=[y, N]=[a, N]$. This implies that $G$ is abelian, which is impossible. Hence $G$ is finite.

(b) Let $G$ be a minimal non-I-group which also a 2-group and suppose that $G$ is infinite. Let $G_{i}$ be the subgroup generated by all elements in $G$ with order dividing $2^{i}$. Suppose that $G_{i}$ is soluble and let $a$ and $b$ be elements of order $2^{i+1}$ not in $G_{i}$. The group $F=\left\langle a G_{i}, b G_{i}\right\rangle$ is generated by two involutions, so it is a dihedral 2-group and in particular is soluble. If $F \notin \mathfrak{T}$, then $F=G / G_{i}$ by 
minimality of $G$ and this implies that $G$ is soluble. If each $F \in \mathfrak{T}$, then each $F$ is abelian and therefore $G_{i+1} / G_{i}$ is abelian and $G_{i+1}$ is soluble. Hence by induction on $j$ each $G_{j}$ is soluble. But $G$ is finitely generated, so $G=G_{j}$ for some $j$ and $G$ is soluble. By (a) $G$ is finite.

This argument shows also that a $\overline{\mathfrak{I}}$-group which is a 2-group is soluble (and hence, by Theorem 6.1.1 of [9], is either abelian or hamiltonian)

(c) Finally let $G$ be as described in the statement of the theorem and let $\left\{\Lambda_{\sigma}, V_{\sigma}: \sigma \in \Sigma\right\}$ be the series in $G$. If $x_{1}, \cdots, x_{m}$ is a set of nontrivial generators for $G$, then $x_{i} \in \Lambda_{\sigma_{i}} \backslash V_{\sigma_{i}}$ for some $\sigma_{i} \in \Sigma$ and if $\sigma=\max \left\{\sigma_{1}, \cdots, \sigma_{m}\right\}, V_{\sigma}<\Lambda_{\sigma}=G$. Let $N=V_{\sigma}$, so that $N \triangleleft G$ and $G / N$ is either locally finite or a 2-group. A factor group of $G$ is

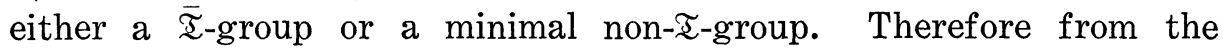
information obtained in (b) and the fact that $G$ is finitely generated we conclude that $G / N$ is finite. Finite $\overline{\mathfrak{T}}$-groups and finite minimal non-T-groups are soluble, so $G / N$ is soluble and $G>G^{\prime}$.

Let $X$ be a finitely generated subgroup of $G^{\prime}$. Since $X \neq G$, $X \in \overline{\mathfrak{I}}$ and therefore $X^{\prime \prime}=X^{\prime \prime \prime}=D$ say, since soluble $\mathfrak{I}$-groups are metabelian ([9], Th. 2.3.1). Suppose that $D \neq 1$. By intersecting the given series in $G$ with $D$, term by term, we obtain a series in $D$ of the same type. Now since $X \in \overline{\mathfrak{I}}$ and each term of the series in $D$ is ascendant or descendant in $D$ and hence in $X$, it follows easily that each term is normal in $X$. Also $X / D$ is a finitely generated metabelian $\mathfrak{I}$-group, so it is either finite or abelian and in either case is certainly finitely presented. Therefore by a well-known principle $D=a_{1}^{X} \cdots a_{n}^{X}$ for some finite subset $\left\{a_{1}, \cdots, a_{n}\right\}$ (see for example [3], p. 421). In precisely the same way as for $G$ we can show that $D>D^{\prime}$. By this contradiction $D=1$ and every finitely generated subgroup of $G^{\prime}$ is metabelian. Hence $G$ is soluble and the result follows by (a).

\section{REFERENCES}

1. K. Doerk, Minimal nicht überauflösbare endliche Gruppen, Math. Zeit. 91 (1966). 198-205.

2. W. Gaschütz, Gruppen in denen das Normalteilersein transitiv ist, J. reine angew. Math. 198 (1957), 87-92.

3. P. Hall, Finiteness conditions for soluble groups, Proc. London Math. Soc. (3) 4 (1954), 419-436.

4. - On non-strictly simple groups, Proc. Cambridge Philos. Soc. 59 (1963), 531-553.

5. A. G. Kuroš, The theory of groups, second ed, New York, Cheìsea, 1960.

6. G. A. Miller and H. C. Moreno, Non-abelian groups in which every subgroup is abelian, Trans. Amer. Math. Soc. 4 (1903), 398-404.

7. T. A. Peng, Finite groups with pronormal subgroups, Proc. Amer. Math. Soc. 20 
(1969), 232-234.

8. L. Rédei, Das schiefe Product in der Gruppentheorie, Comm. Math. Helvetici 20 (1947), 225-264.

9. D. J. S. Robinson, Groups in which normality is a transitive relation, Proc. Cambridge Philos. Soc. 60 (1964), 21-38.

10. - On finitely generated soluble groups, Proc. London Math. Soc. (3) 15 (1965), 508-516.

11. - A note on finite groups in which normality transitive, Proc. Amer. Math. Soc. 19 (1968), 933-939.

12 B. L. van der Waerden, Algebra, 1 Teil, 5 Aufl., Berlin, Springer Verlag, 1960.

Received May 14, 1969.

UNIVERSITY OF ILLINOIS

URBANA, ILLINOIS 



\title{
PACIFIC JOURNAL OF MATHEMATICS
}

\author{
EDITORS
}

\author{
H. ROYDEN \\ Stanford University \\ Stanford, California \\ RICHARD PIERCE \\ University of Washington \\ Seattle, Washington 98105
}

\author{
J. DUGUNDJI \\ Department of Mathematics \\ University of Southern California \\ Los Angeles, California 90007 \\ BASIL GORDON \\ University of California \\ Los Angeles, California 90024
}

\section{ASSOCIATE EDITORS}

E. F. BECKENBACH
B. H. NeumanN

F. WOLF
K. YoshidA

\section{SUPPORTING INSTITUTIONS}

\author{
UNIVERSITY OF BRITISH COLUMBIA \\ CALIFORNIA INSTITUTE OF TECHNOLOGY \\ UNIVERSITY OF CALIFORNIA \\ MONTANA STATE UNIVERSITY \\ UNIVERSITY OF NEVADA \\ NEW MEXICO STATE UNIVERSITY \\ OREGON STATE UNIVERSITY \\ UNIVERSITY OF OREGON \\ OSAKA UNIVERSITY \\ UNIVERSITY OF SOUTHERN CALIFORNIA
}

\author{
STANFORD UNIVERSITY \\ UNIVERSITY OF TOKYO \\ UNIVERSITY OF UTAH \\ WASHINGTON STATE UNIVERSITY \\ UNIVERSITY OF WASHINGTON

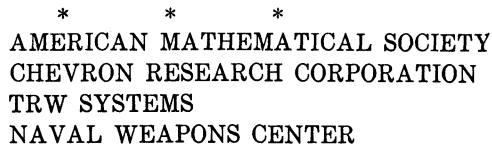

The Supporting Institutions listed above contribute to the cost of publication of this Journal, but they are not owners or publishers and have no responsibility for its content or policies.

Mathematical papers intended for publication in the Pacific Journal of Mathematics should be in typed form or offset-reproduced, double spaced with large margins. Underline Greek letters in red, German in green, and script in blue. The first paragraph or two must be capable of being used separately as a synopsis of the entire paper. It should not contain references to the bibliography. Manuscripts, in duplicate if possible, may be sent to any one of the four editors. Please classify according to the scheme of Math. Rev. 36, 1539-1546. All other communications to the editors should be addressed to the managing editor, Richard Arens, University of California, Los Angeles, California, 90024.

50 reprints are provided free for each article; additional copies may be obtained at cost in multiples of 50 .

The Pacific Journal of Mathematics is published monthly. Effective with Volume 16 the price per volume (3 numbers) is $\$ 8.00$; single issues, $\$ 3.00$. Special price for current issues to individual faculty members of supporting institutions and to individual members of the American Mathematical Society: $\$ 4.00$ per volume; single issues $\$ 1.50$. Back numbers are available.

Subscriptions, orders for back numbers, and changes of address should be sent to Pacific Journal of Mathematics, 103 Highland Boulevard, Berkeley, California, 94708.

PUBLISHED BY PACIFIC JOURNAL OF MATHEMATICS, A NON-PROFIT CORPORATION

Printed at Kokusai Bunken Insatsusha (International Academic Printing Co., Ltd.), 7-17, Fujimi 2-chome, Chiyoda-ku, Tokyo, Japan. 


\section{Pacific Journal of Mathematics}

\section{Vol. 31, No. $3 \quad$ BadMonth, 1969}

George E. Andrews, On a calculus of partition functions .................. 555

Silvio Aurora, A representation theorem for certain connected rings ............ 563

Lawrence Wasson Baggett, A note on groups with finite dual spaces ............. 569

Steven Barry Bank, On majorants for solutions of algebraic differential equations in regions of the complex plane ............................... 573

Klaus R. Bichteler, Locally compact topologies on a group and the corresponding continuous irreducible representations ......................... 583

Mario Borelli, Affine complements of divisors ....................... 595

Carlos Jorge Do Rego Borges, A study of absolute extensor spaces ............. 609

Bruce Langworthy Chalmers, Subspace kernels and minimum problems in Hilbert

spaces with kernel function ...................................... 619

John Dauns, Representation of L-groups and F-rings................. 629

Spencer Ernest Dickson and Kent Ralph Fuller, Algebras for which every

indecomposable right module is invariant in its injective envelope ...........

Robert Fraser and Sam Bernard Nadler, Jr., Sequences of contractive maps and fixed

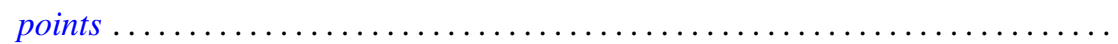

Judith Lee Gersting, A rate of growth criterion for universality of regressive

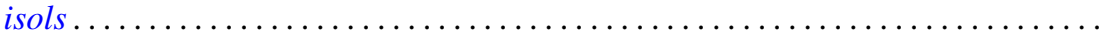

Robert Fred Gordon, Rings in which minimal left ideals are projective ............

Fred Gross, Entire functions of several variables with algebraic derivatives at certain algebraic points

W. Charles (Wilbur) Holland Jr. and Stephen H. McCleary, Wreath products of ordered permutation groups .........................

W. J. Kim, The Schwarzian derivative and multivalence .................. 717

Robert Hamor La Grange, Jr., On $(\mathrm{m}-\mathrm{n})$ products of Boolean algebras ......... 725

Charles D. Masiello, The average of a gauge ........................ 733

Stephen H. McCleary, The closed prime subgroups of certain ordered permutation

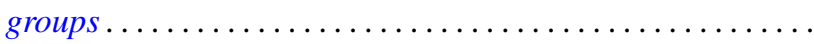

Richard Roy Miller, Gleason parts and Choquet boundary points in convolution measure algebras ...............................

Harold L. Peterson, Jr., On dyadic subspaces ........................ 773

Derek J. S. Robinson, Groups which are minimal with respect to normality being intransitive........................................... 777

Ralph Edwin Showalter, Partial differential equations of Sobolev-Galpern type . . . 787

David Slepian, The content of some extreme simplexes ................... 795

Joseph L. Taylor, Noncommutative convolution measure algebras ............. 809

B. S. Yadav, Contractions of functions and their Fourier series ............... 827

Lindsay Nathan Childs and Frank Rimi DeMeyer, Correction to automorphisms of separable algebras" ....................... 833

Moses Glasner and Richard Emanuel Katz, Correction to: "Function-theoretic degeneracy criteria for Riemannian manifolds".............

Satish Shirali, Correction to: "On the Jordan structure of complex Banach

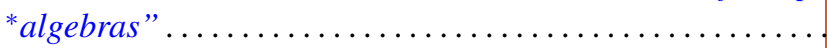

